Pacific Journal of Mathematic 


\section{STRICTLY INCREASING RIESZ NORMS}

L. C. MOORE, JR.

Let $L$ be a Riesz space and $\rho$ a Riesz norm on $L$. Then $\rho$ is said to be strictly increasing if $u, v \in L$ and $0 \leqq u \leqq v$ imply that $\rho(u)<\rho(v)$. We investigate necessary conditions and sufficient conditions that for a given Riesz norm there is an equivalent strictly increasing Riesz norm. A necessary condition is that the Riesz space possess the countable sup property. A sufficient condition is that the given norm be an (A, ii) norm. Finally, we investigate the relationship between the existence of strictly increasing Riesz norms and the Souslin hypothesis.

For reference, we list here several definitions. If $\rho$ is a Riesz norm on a Riesz space $L$, then $\rho^{*}$ is the dual norm on the Riesz space of $\rho$-bounded linear functionals. A Riesz norm $\rho$ is said to have property (A, ii) if whenever $\left\{u_{\lambda}\right\}$ is directed downwards in $L$ and $\inf _{2} u_{2}=0$, then $\inf _{\lambda} \rho\left(u_{\lambda}\right)=0$. An order bounded linear functional $\varphi$ on $L$ is said to be an integral if $\left\{u_{n}\right\}$ is a sequence in $L$ and $u_{n} \downarrow 0$ implies $\inf _{n}\left|\varphi\left(u_{n}\right)\right|=0$. If whenever $\left\{u_{\lambda}\right\}$ is a set in $L$ which is directed downwards and $\inf _{\lambda} u_{\lambda}=0$, then $\inf _{\lambda}\left|\varphi\left(u_{\lambda}\right)\right|=0$, we say that $\varphi$ is a normal integral. If $\rho$ is a Riesz norm on $L$, then $L_{\rho, *}^{*}$ is the Riesz space of all $\rho$-bounded integrals and $L_{\hat{\rho}, n}^{*}$ is the Riesz space of all $\rho$ bounded normal integrals. A Riesz space $L$ is said to have the countable sup property if every nonempty subset $D$ of $L$ possessing a supremum contains an at most countable subset having the same supremum as $D$.

Luxemburg discussed the countable sup property in [3] and, in the case that the Riesz space is Archimedean, gave a number of equivalent conditions (Theorem 6 of [3]). In addition he showed that if $L$ is Dedekind $\sigma$-complete and possesses a strictly increasing Riesz norm, then $L$ has the countable sup property (Theorem 10 of [3]). In a private communication, Luxemburg asked whether the theorem is true if the condition that $L$ be Dedekind $\sigma$-complete is dropped. The answer is yes and is the content of Corollary 2 of this paper. That an (A, ii) norm has an equivalent strictly increasing Riesz norm (Theorem 5) was suggested by Luxemburg, and, in the case that $L$ is countably generated, it appears in [4].

I wish to express my gratitude to C. Ward Henson for many valuable suggestions in the preparation of this paper.

2. Necessary conditions and sufficient conditions. If a Riesz space $L$ with Riesz norm $\rho$ possesses a $\rho$-bounded strictly positive 
linear functional $\varphi$, then

$$
q(f)=\rho(f)+\varphi(|f|) \quad \text { for all } f \in L
$$

defines a strictly increasing Riesz norm $q$ on $L$ such that $q$ is equivalent to $\rho$. On the other hand if $L$ is countably generated, then if $L$ possesses a strictly increasing Riesz norm it must possess a bounded strictly positive linear functional.

THEOREM 1. If $L$ is a Riesz space which is countably generated and $\rho$ is a Riesz norm on $L$, then there is a srictly increasing Riesz norm on $L$ equivalent to $\rho$ if and only if $L$ possesses a strictly positive $\rho$-bounded linsar functional.

Proof. As we have noted, if there is a strictly positive $\rho$ bounded linear functional on $L$, then there is a strictly increasing Riesz norm on $L$ which is equivalent to $\rho$.

Now to show the converse, assume first that $L$ has a strictly increasing Riesz norm $q$ which is equivalent to $\rho$ and a weak unit $w \in L^{+}$. Then by the Hahn-Banach Theorem there is a $q$-bounded linear functional $\varphi$ such that $q^{*}(\varphi)=1$ and $\varphi(w)=q(w)$. Further, we may assume that $\varphi \geqq 0$. Let $A_{w}$ be the principal ideal in $L$ generated by $w$. Then if $0 \leqq v \in A_{w}$, we have that $v \leqq \lambda w$ for some real positive $\lambda$. But since $q$ is strictly increasing,

$$
0 \leqq \varphi(\lambda w-v) \leq q(\lambda w-v)<q(\lambda w)=\varphi(\lambda w) .
$$

Hence $\varphi(\lambda w)-\varphi(v) \geqq \varphi(\lambda w)$ and $\varphi(v)>0$, i.e., $\varphi$ is strictly positive on $A_{w}$. Now if $z \in L^{+}$and $z \neq 0$, then for some $v \in A_{w}$, we have $0 ફ v \leqq z$. Hence $\varphi(z) \geqq \varphi(v)>0$. Thus $\varphi$ is strictly positive on $L$.

If $L$ does not have a weak unit, then there is a countable set $\left\{w_{n}: n=1,2, \cdots\right\}$ in $L^{+}$such that if $0 ફ z \in L$, there are an integer $n$ and an element $v \in L^{+}$such that $0 \leqq v \leqq \inf \left(z, w_{n}\right)$. As above, we select positive bounded linear functionals $\varphi_{n}$ such that $q^{*}\left(\varphi_{n}\right)=1$ and $\varphi_{n}\left(w_{n}\right)=q\left(w_{n}\right)$ for $n=1,2, \cdots$. Then $\varphi=\sum_{n=1}^{\infty}\left(1 / 2^{n}\right) \varphi_{n}$ is the desired strictly positive bounded linear functional.

In Example 6 we see an example of a Riesz space which is not countably generated, which possesses a strictly increasing Riesz norm, but which does not possess a strictly positive bounded linear functional.

We have the following corollary to Theorem 1 , which generalizes Theorem 10 of [3].

CoROLlary 2. If a Riesz space $L$ possesses a strictly increasing Riesz norm, then $L$ has the countable sup property. 
Proof. If $L$ possesses a strictly increasing Riesz norm, then $L$ is Archimedean. Thus in order to show that $L$ has the countable sup property, it is sufficient to show that every disjoint order bounded set of positive elements is at most countable. (See Theorem 6 of [3].) But this is evident since by Theorem 1 every principal ideal in $L$ possesses a strictly positive linear functional.

In Example 4, we see that the countable sup property is not sufficient for the existence of a strictly increasing Riesz norm, even for an Archimedean Riesz space with a strong unit.

We now give a sufficient condition for the existence of a strictly increasing Riesz norm equivalent to a given Riesz norm. This result generalizes Corollary 35.3 of [4].

Theorem 3. If $L$ has the countabls sup property, $L$ is countably generated, and $L$ possesses a Ricsz norm $\rho$ such that $L_{p, c}^{*}$ separates points in $L$, then $L$ possesses a $\rho$-bounded strict'y positive norma! integral. Hence, there is a strictly increasing Ricsz norm $q$ on $L$ which is equiva? ent to $\rho$.

Proof. Note that since $L$ has the countable sup property, we have $L_{\rho, c}^{*}=L_{\rho, n}^{*}$.

We show first that if $w \in L^{+}$, then there is a positive normal integral $\varphi_{i} \in L_{i, n}^{*}$ which is strictly positive on the principal ideal $A_{w}$ generated by $w$. For each $\varphi \in L_{\rho, n}^{*}$ let $B_{\varphi}=\left\{f \in A_{w}: \varphi(|f|)=0\right\}$. Let $\mathscr{S}=\left\{B_{\varphi}: \varphi \in L_{\rho, n}^{*}\right.$ and $\left.\rho^{*}(\varphi)=1\right\}$. Then if $B_{\varphi} \in \mathscr{P}$, we have that $B_{\varphi}$ is a band in $A_{w}$, since $\varphi$ is a normal integral. Now consider $\mathscr{B}$ to be partially ordered by containment. We want to show that $\mathscr{B}$ has a minimal element $B_{\varphi_{0}}$. Then since $L_{o, n}^{*}$ separates points in $L$, we must have $B_{\varphi_{0}}=\{0\}$ and $\varphi_{0}$ must be strictly positive on $A_{w}$.

To show this let $\mathscr{C}$ be a nonempty subset of $\mathscr{B}$ which is wellordered by $\supseteq$. We want to show that $\mathscr{C}$ has a lower bound in $\mathscr{B}$. If $\mathscr{C}$ has an upper bound $B_{\psi}$ in $\mathscr{C}$, let $\mathscr{C}^{\prime}=\mathscr{C} \sim\left\{B_{\psi}\right\}$. Then for every $B \in \mathscr{C}^{\prime}$, there is a predecessor $B_{\tilde{o}} \in \mathscr{C}$ such that $B_{\tilde{\ddots}} \supsetneq B$ and if $B_{0} \in \mathscr{C}$ and $B_{\theta} \supseteq B$, then $B_{\theta} \supseteq B_{\tilde{c}}$. Since $L$ is Archimedean, $A_{w}$ is Archimedean also; and for each $B_{\varphi} \in \mathscr{C}^{\prime}$ there exists $0 ફ u_{\varphi}$ in $B_{\widetilde{\varphi}} \cap B_{\varphi}$ such that $v_{\varphi} \leq w$, where $B_{c}=\left\{f \in A_{w}: \inf (|f|),|g|\right)=0$ all $\left.g \in B_{\varphi}\right\}$. Then $\left\{v_{\varphi}: B_{\varphi} \in \mathscr{C}^{\prime}\right\}$ is a disjoint order-bounded set in $L$. Since $L$ has the countable sup property, it is at most countable. It follows that $\mathscr{C}^{\prime}$ and $\mathscr{C}$ are at most countable. If $\mathscr{C}$ is finite, $\mathscr{C}$ has a lower bound. If $\mathscr{C}=\left\{B_{\varphi_{n}}: n=1,2, \cdots\right\}$, let $\pi=\sum_{n=1}^{\infty}\left(1 / 2^{n}\right) \varphi_{n}$ and $\varphi=\left(1 / \rho^{*}(\pi)\right) \pi$. Then $B \in \mathscr{B}$ and $B_{\varphi}$ is a lower bound for $\mathscr{C}$. Now by Zorn's Lemma, $\mathscr{B}$ has a minimal element.

Finally, if $\left\{w_{k}: k=1,2, \cdots\right\}$ is a countable set of positive genera- 
tors for $L$, for each $k$ we select a positive bounded normal integral $\varphi_{k}$ which is strictly positive on $A_{w_{k}}$ and such that $\rho^{*}\left(\varphi_{k}\right)=1 / 2^{k}$. on $L$.

Then $\rho=\sum_{k=1}^{\infty} \varphi_{k}$ is a strictly positive bounded normal integral

Example 4. Let $X$ be an uncountable set and $L$ the Riesz space of bounded real-valued functions $f$ such that $\{x \in X: f(x) \neq 0\}$ is at most countable. Then $L$ has the countable sup property but $L$ is not countably generated. If $\rho$ is the uniform norm, $L_{\rho, n}^{*}$ separates points in $L$.

We show that $L$ cannot possess a strictly increasing Riesz norm. Let $q$ be a Riesz norm on $L$. Let $\alpha=\sup \left\{q\left(\chi_{A}\right): A\right.$ is an at most countable subset of $X\}$. Pick countable subsets $\left\{A_{n}: n=1,2, \cdots\right\}$ such that $q\left(\chi_{A_{i n}}\right) \uparrow \alpha$. Let $A_{0}=\bigcup_{n=1}^{\infty} A_{n}$ and $x_{0} \in X \sim A_{0}$. If $A=A_{0} \cup\left\{x_{0}\right\}$, then $\alpha \leqq q\left(\chi_{A_{0}}\right) \leqq q\left(\chi_{A}\right) \leqq \alpha$ and $q\left(\chi_{A_{0}}\right)=q\left(\chi_{A}\right)$. Thus $q$ is not strictly increasing.

In the case that $\rho$ is an $(\mathrm{A}, \mathrm{ii})$ norm on $L$, the condition in Theorem 3 that $L$ be countably generated can be dropped.

THEOREM 5. If $L$ possesses an (A, ii) norm $\rho$, then $L$ possesses a strictly increasing Riesz norm $q$ which is equivalent to $\rho$.

Proof. Since $L$ possesses an (A, ii) norm $\rho, L$ has the countable sup property (Theorem 33.2 in [4]). Let $\left\{u_{\lambda}: \lambda \in \Lambda\right\}$ be a maximal orthogonal subset of positive elements in $L$. If $\Lambda$ is finite or countable, the result follows from Theorem 3. Thus we may assume $A$ is uncountable.

For each $\lambda \in \Lambda$ let $A_{\lambda}$ be the principal ideal in $L$ generated by $u_{\lambda}$. By Theorem 3 for each $\lambda$ there exists $\varphi_{\lambda} \in A_{\lambda, \rho}^{*}$ such that $\varphi_{\lambda}$ is strictly positive on $A_{\lambda}$ and $\mid \varphi_{\lambda}(f) \leqq \rho(f)$ for all $f \in A_{\lambda}$.

Now for each $\lambda$ we define a Riesz seminorm $q_{\lambda}$ on $L$ by setting

$$
q_{\lambda}(f)=\sup \left\{\varphi_{\lambda}(u): 0 \leqq u \leqq|f|, u \in A_{\lambda}\right\}
$$

for all $f \in L$. It is easy to see that for each $\lambda$ we have $q_{\lambda}(f) \leqq \rho(f)$ and that if $0 \neq f \in L$, then for some $\lambda \in \Lambda$ we have $q_{\lambda}(f)>0$. On the other hand, since $L$ has the countable sup property, for any $f \in L$ the set $\left\{\lambda \in \Lambda: q_{2}(f)>0\right\}$ is at most countable.

Now if $0 \leqq v \in L$, then $0=\inf \left\{v-\sum_{\lambda \in F} u_{\lambda}: F\right.$ is a nonempty finite subset of $A$ and for each $\lambda \in F$ we have $0 \leqq u_{\lambda} \in A_{\lambda}$ and $\left.u_{\lambda} \leqq v\right\}$. Since $\rho$ is an (A, ii) norm, for every real $\varepsilon>0$ there is a finite subset $F$ of $\Lambda$ and an element $u_{\lambda} \in A_{\lambda}^{+}$for each $\lambda \in F$ such that $\sum_{\lambda \in F} u_{\lambda} \leqq v$ and $\rho\left(v-\sum_{\lambda \in F} u_{\lambda}\right)<\varepsilon$. So if $\lambda^{\prime} \in \Lambda \sim F$ and $u \in A_{\lambda}$ such that $0 \leqq u \leqq v$, then $u \leqq v-\sum_{\lambda \in F} u_{\lambda}$ and $\rho(u)<\varepsilon$. It follows that for any $f \in L$ and $\varepsilon>0$ there are only finitely many $\lambda$ 's such that $q_{\lambda}(f) \geqq \varepsilon$. 
We now define a sequence of Riesz norms on $L$. Let $q_{n}(f)=$ $\sup \left\{(1 / n) \sum_{\lambda \in F} q_{\lambda}(f): F \subseteq \Lambda\right.$ and $F$ has exactly $n$ elements $\}$ for $f \in L$ and $n=1,2, \cdots$. For each $n$ it is clear that $q_{n}$ is a Riesz norm and $q_{n}(f) \leqq \rho(f)$ for all $f \in L$. Let

$$
q_{\infty}(f)=\sum_{n=1}^{\infty} \frac{1}{2^{n}} q_{n}(f)
$$

for all $f \in L$. Then again $q_{\infty}$ is a Riesz norm and $q_{\infty}(f) \leqq \rho(f)$ for all $f \in L$.

Now suppose $u, v \in L^{+}$and $v-u=w \supsetneqq 0$. Then for some $\lambda_{0}$ we have $q_{\lambda_{0}}(w)>0$. It follows that there is $0 \leqq z \in A_{\lambda_{0}}$ such that $z \leqq w$ and $\varphi_{\lambda_{0}}(z)>0$. Now if $0 \leqq y \leqq u$ and $y \in A_{\lambda_{0}}$, then $y+z \leqq v$. Thus $\varphi_{\lambda_{0}}(y)+\varphi_{\lambda_{0}}(z)<q_{\lambda_{0}}(v)$ and $q_{\lambda_{0}}(u)<q_{\lambda_{0}}(v)-\varphi_{\lambda_{0}}(z)$, i.e., $q_{\lambda_{0}}(v)-$ $q_{\lambda_{0}}(u)>0$. Now pick a real positive $\delta$ such that $q_{\lambda_{0}}(u) \leqq \delta \leqq q_{\lambda_{0}}(v)$. Then $\left\{\lambda \in \Lambda: q_{\lambda}(v)>\delta\right\}$ is finite say $\left\{\lambda_{0}, \lambda_{1}, \ldots, \lambda_{n}\right\}$. Clearly $q_{n+1}(v)=$ $(1 /(n+1)) \sum_{k=0}^{n} q_{\lambda_{k}}(v)$. On the other hand, $\left\{\lambda: q_{\lambda}(u)>\delta\right\} \subseteq\left\{\lambda_{1}, \cdots, \lambda_{n}\right\}$. So

$$
q_{n+1}(u) \leqq \frac{1}{n+1}\left[\delta+\sum_{k=1}^{n+1} q_{\lambda_{k}}(v)\right]<q_{n+1}(v) .
$$

Thus $q_{\infty}(u)<q_{\infty}(v)$ and $q_{\infty}$ is strictly increasing. Now $q=p+q_{\infty}$ is the desired Riesz norm.

However, as we see in Example 6, if $\rho$ is an (A, ii) norm on a Riesz space $L$ which is not countably generated, $L$ need not have a strictly positive linear functional. (This example is given on page 512 of [4].)

EXAMPLe 6 . Let $X$ be an uncountable set and $L$ the Riesz space of all real-valued functions $f$ on $X$ such that for every $\varepsilon>0$ the set $\{x \in X:|f(x)| \geqq \varepsilon\}$ is finite. Then the sup norm is an (A, ii) norm on $L$, but $L$ does not possess a strictly positive linear functional.

3. The ramification property. As we have seen in Theorem 1, the existence of strictly increasing Riesz norms is related to the existence of strictly positive linear functionals. The existence of these functionals is related, in turn, to the existence of strictly positive finitely additive measures on Boolean algebras and to the Souslin hypothesis. We investigate these relations below.

Horn and Tarski [2] define a subset $S$ of a Boolean algebra to be a ramification set if: and,

(i) for every $x$ and $y$ in $S$ either $\inf (x, y)=0$ or $x \leqq y$ or $y \leqq x$,

(ii) for every $x$ in $S$ the set $\{y: y \in S$ and $y \geqq x\}$ is well-ordered by the relation $\geqq$. 
Recall that a Boolean algebra $B$ satisfies the countable chain condition if every set of orthogonal elements in $B$ is at most countable.

In the paper cited above [2], Horn and Tarski prove the following results.

I. If a Boolean algebra $B$ possesses a strictly positive finitely additive measure, then every ramification set in $B$ is at most countable.

II. If every ramification set in a Boolean algebra $B$ is at most countable, then $B$ satisfies the countable chain condition.

In addition, Horn and Tarski show that the following statements are equivalent.

A. Every Boolean algebra satisfying the countable chain condition also has the property that every ramification set is at most countable.

B. The Souslin hypothesis holds.

We extend the concept of a ramification set to Riesz spaces. Let $L$ be a Riesz space. A subset $S$ of $L^{+}$is said to be a ramification set if: and,

(i ) for every $x$ and $y$ in $S$ either $\inf (x, y)=0$ or $x \leqq y$ or $y \leqq x$,

(ii) for every $x$ in $S$ the set $\{y: y \in S$ and $y \geqq x\}$ is well-ordered by the relation $\geqq$.

The Riesz space $L$ is said to satisfy the ramification property if every order-bounded ramification set is at most countable.

Note that if $L$ is an Archimedean Riesz space satisfying the ramification property then every order-bounded orthogonal subset of positive elements is a ramification set and hence countable. Thus for Archimedean Riesz spaces the ramification property implies the countable sup property. (See Theorem 6 in [3].)

THEOREM 7. If a Riesz space $L$ possesses a strictly increasing Riesz norm, then $L$ satisfies the ramification property.

Proof. Suppose $\rho$ is a strictly increasing Riesz norm on $L$. Let $S$ be a ramification set in $L^{+}$and assume $S$ is bounded above by $e \in L^{+}$.

Now for each $u \in S$ we have $\left\{v: v \in S\right.$ and $\left.v \geqq v_{u}\right\}$ is well-ordered. Thus $\{\rho(v): v \in S$ and $v \geqq u\}$ is a well-ordered set of real numbers. Since $\rho$ is strictly increasing, it follows that $\{v: v \in S$ and $v \geqq u\}$ is at most countable for each $u \in S$.

For each $u \in S$, let

$$
\alpha(u)=\inf \{\rho(v): v \in S \text { and } v \leqq u\}
$$

and $S_{0}=\{u \in S: \alpha(u)=\rho(u)\}$. If $u$ and $u$ are distinct elements of $S_{0}$, we cannot have $w \leqq u$, since then $\rho(u)>\rho(w) \geqq \alpha^{\prime}(u)$. Similarly we cannot have $u \leqq w$. Thus $S_{\text {v }}$ is an order-bounded orthogonal set. By 
Corollary $2 L$ has the countable sup property, so, $S_{0}$ is at most countable. Now let $T_{0}=\left\{v \in S: v \geqq u\right.$ for some $\left.u \in S_{c}\right\}$. Then

$$
T_{0}=\bigcup_{u \in S_{0}}\{v: v \in S \text { and } v \geqq u\}
$$

and $T_{0}$ is at most countable. Let $T=S \sim T_{0}$.

Now for $n=1,2, \ldots$, let $S_{n}$ be a maximal orthogonal subset of the set $\{u \in T: \rho(u)-\alpha(u)<1 / n\}$. Let $T_{n}=\{v \in S: v \geqq u$ for some $\left.u \in S_{n}\right\}$ for $n=1,2, \cdots$. Then, as above, both $S_{n}$ and $T_{n}$ are at most countable for each $n$.

Now if $z \in T$ we have $\rho(z)-\alpha(z)>0$. Pick $n$ such that $\rho(z)-$ $\alpha(z)>1 / n$ and $w \in S$ such that $w \leqq z$ and $\rho(w)-\alpha(z)<1 / n$. Then since $\alpha(w) \geqq \alpha(z)$, we have

$\rho(w)-\alpha(w) \leqq \rho(w)-\alpha(z)<1 / n$. Now by the maximality of $S_{n}$ for some $u \in S_{n}$ we have $\inf (u, w)>0$. It follows that $\inf (u, z)>0$. Hence, either $u \leqq z$ or $z \leqq u$. However, if $z \leqq u$, then $\rho(u)-\alpha(u) \geqq$ $\rho(z)-\alpha(z)>1 / n$, which is impossible. Thus $u \leqq z$ and $z \in T_{n}$. So $S=\bigcup_{n=0}^{\infty} T_{n}$ and $S$ is at most countable. This shows that $L$ has the ramification property.

Lemma 8. If $W$ is an order-bounded subset of an Archimedsan Riesz space $L$ satisfying the countable sup property and $W$ is wellorderd by $\geqq($ or by $\leqq)$, then $W$ is at most countable.

Proof. We may assume that $W \subseteq L^{+}, W$ is well-ordered by $\geqq$, and $W$ is bounded above by $e \in L^{+}$. If $W$ has a maximal element $u_{\nu}$, let $W^{\prime}=W \sim\left\{u_{0}\right\}$. If not, let $W^{\prime}=W$. We may assume that $W^{\prime}$ is infinite.

Now for every $u \in W^{\prime}$ there exists $\tilde{u} \in W$ such that $u \lessgtr \widetilde{u}$ and if $v \in W$ and $u \leqq v$ then $\widetilde{u} \leqq v$. For each $u \in W^{\prime}$ let $w_{u}=\widetilde{u}-u$. Then for every nonempty finite subset $F$ of $W^{\prime}$ we have $\sum_{u s F} w_{u} \leqq \ell$. Since $L$ is Archimedean and satisfies the countable sup property, the set $\mathscr{S}=\left\{\sum_{u \in F} w_{u}: F\right.$ is a nonempty finite subset of $\left.W\right\}$ has a countable subset $\mathscr{C}$ with the same upper bounds. (Again see Theorem 6 in [3].) It follows that there exists a countable collection $\left\{v_{1}, u_{2}, \cdots\right\}$ of distinct elements of $W^{\prime}$ such that $v$ is an upper bound for $\mathscr{S}$ if and only if $\sum_{k=1}^{n} w_{u_{k}} \leqq v$ for $n=1,2, \cdots$.

If for some $z \in W^{\prime}$ we have $z \neq u_{k}$ for any $k$, then we have

$$
0<\sum_{k=1}^{n} w_{u_{k}}+w_{z} \leqq e \quad \text { or } \quad \sum_{k=1}^{n} w_{u_{k}} \leqq e-w_{z}
$$

for all $n$. But then for all $n$

$$
0<\sum_{k=1}^{n} w_{u_{k}}+w_{z} \leqq e-w_{z} \quad \text { or } \quad 0 \leqq e-2 w_{z} \text {. }
$$


Continuing in this manner, we see that $n w_{z} \leqq e$ for all $n$, which implies that $w_{z}=0$. But this is impossible. So $\left\{u_{1}, u_{2}, \cdots\right\}=W^{\prime}$ and both $W^{\prime}$ and $W$ are countable.

If $B$ is a Boolean algebra, we apply the Stone Representation Theorem and consider $B$ as the Boolean algebra of closed-open sets of a totally disconnected compact Hausdorff space $X(B)$. We associate with $B$ the Riesz space $L(B)$ of all real-valued functions $f$ defined on $X(B)$ such that the range of $f$ is a finite set and for any real $\alpha$ the set $\{x \in X(B): f(x)=\alpha\}$ is closed-open set. It is clear that for any Boolean algebra $B$, the space $L(B)$ is an Archimedean Riesz space with a strong unit.

Theorem 9. Let $B$ be a Boolean algebra. Then:

(i ) $L(B)$ has the countable sup property if and only if $L$ satisfies the countable chain condition.

(ii) $L(B)$ has the ramification property if and only if every ramification set in $B$ is at most countable.

(iii) $L(B)$ possesses a strictly increasing Riesz norm if and only if $B$ possesses a strictly positive finitely additive measure.

Proof. (i) The proof of (i) is evident once we recall that, for an Archimedean Riesz space, the countable sup property is equivalent to the condition that every order-bounded orthogonal subset of $L^{+}$ is at most countable.

(ii) It is clear that if $L(B)$ has the ramification property then every ramification set in $B$ is at most countable.

We now assume that every ramification set in $B$ is at most countable. Then $B$ satisfies the countable chain condition and by (i) $L(B)$ has the countable sup property. Let $S$ be an order-bounded ramification set in $L^{+}$. For every $u \in S$ let $X_{u}=\{x \in X(B): u(x)>0\}$. Then for each $u \in S$ the set $X_{u}$ is a closed-open set in $X(B)$. Now if $u, v \in S$ and $X_{u} \cap X_{v} \neq 0$, then $\inf (u, v) \neq \varnothing$. Thus we have either $u \leqq v$ or $v \leqq u$, i.e., $X_{u} \subseteq X_{v}$ or $X_{v} \subseteq X_{u}$. In addition, for any $u \in S$, we see that since $\{v \in S: v \geqq u\}$ is well-ordered by $\geqq$ then $\left\{X_{v}: v \in S\right.$ and $\left.X_{v} \supseteq X_{u}\right\}$ is well-ordered by $\supseteq$. It follows that $\left\{X_{u}: u \in S\right\}$ is a ramification set in $B$ and so is at most countable. In addition, for any $u \in S$ the set $\left\{v: X_{v}=X_{u}\right\}$ is well-ordered by $\geqq$. Thus by Lemma 8, each of the sets $\left\{v: X_{v}=X_{u}\right\}$ is at most countable. Hence, $S$ is at most countable and $L(B)$ has the ramification property.

(iii) Suppose $L(B)$ possesses a strictly increasing Riesz norm. Then by Theorem $1, L$ possesses a strictly positive linear functional $\varphi$. If $O$ is a closed-open set in $X(B)$, let $m(O)=\varphi\left(\chi_{0}\right)$. Then $m$ is a strictly positive finitely additive measure on $B$.

On the other hand, let $m$ be a strictly positive finitely additive 
measure on $B$. Then if $f \in L(B)$ and $f=\sum_{k=1}^{n} r_{k} \chi_{o_{k}}$ where each $O_{k}$ is a closed-open set and the $r_{k}$ 's are distinct nonzero real numbers, let $\varphi(f)=\sum_{k=1}^{n} r_{k} m\left(O_{k}\right)$. The linear functional $\varphi$, defined in this way, is strictly positive and

$$
\rho(f)=\varphi(|f|) \text { for all } f \in L(B)
$$

defines a strictly increasing Riesz norm $\rho$ on $L(B)$.

Recall that the Souslin hypothesis states that if

(i ) $X$ is a totally ordered set without first and last elements,

(ii) with the interval topology $X$ is connected, and,

(iii) each collection of disjoint intervals is at most countable, then $X$ is separable and, hence, homeomorphic to the real line.

A set $T$ with a partial ordering $\leqq$ is called a tree if for each $t \in T$ we have that the set $\{s \in T: s \leqq t\}$ is well-ordered by $\leqq$. A Souslin tree is a tree $T$ of cardinality $\aleph_{1}$ such that each totally ordered subset of $T$ is at most countable and each pairwise unordered subset of $T$ is at most countable. The Souslin hypothesis is equivalent to the statement that there are no Souslin trees. (See [5] for a summary of recent results concerning the Souslin hypothesis.)

THEOREM 10. The following statements are equivalent.

(i) Every Archimedean Riesz space possessing the countable sup property possesses the ramification property.

(ii) The Soustin hypothesis holds.

Proof. (ii) implies (i). We assume that $L$ is an Archimedean Riesz space possessing the countable sup property and that the Souslin hypothesis holds. Let $S$ be a ramification set in $L^{+}$which is bounded above by $e \in L^{+}$. We shall show that if $S$ is uncountable then $S$ contains a Souslin tree, contradicting the Souslin hypothesis.

Now $S$ is clearly a tree under $\geqq$. If $A$ is a pairwise unordered subset of $S$, then $A$ is an order-bounded orthogonal subset of $L^{+}$. Hence $A$ is at most countable by the countable sup property. Now suppose that $B$ is a totally ordered subset of $S$. Then $B$ is a well-ordered, order-bounded subset of an Archimedean Riesz space. So $B$ is at most countable by Lemma 8 .

If $S$ is uncountable, then any subset of cardinality $W_{1}$ is a Souslin tree under $\geqq$. Thus $S$ is at most countable and $L$ possesses the ramification property.

(i) implies (ii). Now suppose that every Archimedean Riesz space possessing the countable sup property also possesses the ramification property. Then by Theorem 9 we see that every Boolean algebra satisfying the countable chain condition also has the property that every ramification set is at most countable. Thus by the result of 
Horn and Tarski stated above, the Souslin hypothesis must hold.

ExAmPLe 11. It is not true that an Archimedean Riesz space $L$ with a strong unit and such that $L$ possesses the ramification property must possess a strictly increasing Riesz norm. Such a Riesz space is $L(B)$ where $B$ is a Boolean algebra which satisfies the condition that every ramification set at most countable but $B$ does not possess a strictly positive finitely additive measure. An example of a Boolean algebra with these properties was constructed by Haim Gaifman in [1]. Indeed, in Gaifman's example, the Boolean algebra $B$ satisfies. the condition below.

(*) There are sets $B_{1}, B_{2}, \cdots$, in $B$ such that for each $n$ every orthogonal subset of $B_{n}$ contains at most $n$ elements and $B=\bigcup_{n=1}^{\infty} B_{n}$.

Horn and Tarski have shown [2] that for a Boolean algebra $B$ the condition (*) implies that every ramification set is at most countable and is implied by the existence of a strictly positive finitely additive measure on $B$. If $B$ is the example of Gaifman, it is easy to see that the Riesz space $L(B)$ satisfies the condition that there are sets $L_{1}, L_{2}, \cdots$ in $L$ such that for each $n$ every orthogonal subset of $L_{n}$ contains at most $n$ elements and $L=\bigcup_{n=1}^{\infty} L_{n}$.

\section{REFERENCES}

1. H. Gaifman, Concerning measures on Boolean algebras, Pacific J, Math., 14 (1964), 61-73.

2. A. Horn and A. Tarski, Measures in Boolean algebras, Trans. Amer. Math. Soc., 64 (1948), 467-497.

3. W. A. J. Luxemburg, On some order properties of Riesz spaces and their relations, Arch. Math., 19 (1968), 488-493.

4. W. A. J. Luxemburg and A. C. Zaanen, Notes on Banach functions spaces, Proc. Acad. Sci. Amsterdam; Note X, A67 (1964), 493-506, Note XI, A67 (1964), 507-518.

5. M. E. Rudin, Souslin's conjecture, Amer. Math. Monthly, 76 (1969), 1113-1119.

Received July 15, 1970.

DUKE UNIVERSITY 


\section{PACIFIC JOURNAL OF MATHEMATICS}

\section{EDITORS}

H. SAMELSON

Stanford University

Stanford, California 94305

C. R. Новву

University of Washington

Seattle, Washington 98105
J. DUGundJI

Department of Mathematics

University of Southern California

Los Angeles, California 90007

RICHARD ARENS

University of California

Los Angeles, California 90024

\section{ASSOCIATE EDITORS}
E. F. BECKENBACH
B. H. NeumanN
F. WOLF
K. YOSHIDA

\section{SUPPORTING INSTITUTIONS}
UNIVERSITY OF BRITISH COLUMBIA
CALIFORNIA INSTITUTE OF TECHNOLOGY
UNIVERSITY OF CALIFORNIA
MONTANA STATE UNIVERSITY
UNIVERSITY OF NEVADA
NEW MEXICO STATE UNIVERSITY
OREGON STATE UNIVERSITY
UNIVERSITY OF OREGON
OSAKA UNIVERSITY
UNIVERSITY OF SOUTHERN CALIFORNIA
STANFORD UNIVERSITY
UNIVERSITY OF TOKYO
UNIVERSITY OF UTAH
WASHINGTON STATE UNIVERSITY
UNIVERSITY OF WASHINGTON
AMERICAN MATHEMATICAL SOCIETY CHEVRON RESEARCH CORPORATION TRW SYSTEMS
NAVAL WEAPONS CENTER 


\section{Pacific Journal of Mathematics}

\section{Vol. 37, No. $1 \quad$ January, 1971}

Gregory Frank Bachelis and Haskell Paul Rosenthal, On unconditionally

converging series and biorthogonal systems in a Banach space .........

Richard William Beals, On spectral theory and scattering for elliptic

operators with singular potentials .........................

J. Lennart (John) Berggren, Solvable and supersolvable groups in which every element is conjugate to its inverse ........................ 21

Lindsay Nathan Childs, On covering spaces and Galois extensions ..........

William Jay Davis, David William Dean and Ivan Singer, Multipliers and

unconditional convergence of biorthogonal expansions..............

Leroy John Derr, Triangular matrices with the isoclinal property ............

Paul Erdős, Robert James McEliece and Herbert Taylor, Ramsey bounds for

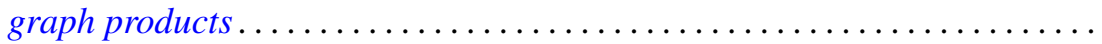

Edward Graham Evans, Jr., On epimorphisms to finitely generated

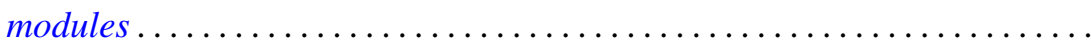

Hector O. Fattorini, The abstract Goursat problem ................. 51

Robert Dutton Fray and David Paul Roselle, Weighted lattice paths .........

Thomas L. Goulding and Augusto H. Ortiz, Structure of semiprime $(p, q)$

radicals ...........................................

E. W. Johnson and J. P. Lediaev, Structure of Noether lattices with join-principal maximal elements ....

David Samuel Kinderlehrer, The regularity of minimal surfaces defined over

slit domains

Alistair H. Lachlan, The transcendental rank of a theory. .

Frank David Lesley, Differentiability of minimal surfaces at the boundary ...

Wolfgang Liebert, Characterization of the endomorphism rings of divisible torsion modules and reduced complete torsion-free modules over complete discrete valuation rings....

Lawrence Carlton Moore, Strictly increasing Riesz norms.

Raymond Moos Redheffer, An inequality for the Hilbert transform ...

James Ted Rogers Jr., Mapping solenoids onto strongly self-entwined,

circle-like continua..........................

Sherman K. Stein, B-sets and planar maps ................... 217

Darrell R. Turnidge, Torsion theories and rings of quotients of Morita

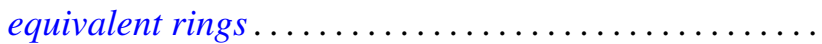

Fred Ustina, The Hausdorff means of double Fourier series and the principle of localization ................................

Stanley Joseph Wertheimer, Quasi-compactness and decompositions for arbitrary relations.

Howard Henry Wicke and John Mays Worrell Jr., On the open continuous images of paracompact $\check{C}$ ech complete spaces... 